\title{
The competition between incoming and outgoing fluxes in an elliptic problem
}

\author{
Jorge García-Melián ${ }^{1}$, Julio D. Rossin ${ }^{2}$ and Antonio SuÁrez ${ }^{3}$, \\ 1. Dpto. de Análisis Matemático, Universidad de La Laguna, \\ C/. Astrofísico Francisco Sánchez s/n, 38271 - La Laguna, SPAIN,
}

2. Facultad de Ciencias Exactas y Naturales, Universidad de Buenos Aires, 1428 - Buenos Aires, ARGENTINA

Instituto de Matemáticas y Física Fundamental, CSIC, C/. Serrano 123, 28006 - Madrid, SPAIN

3. Dpto. de Ecuaciones Diferenciales y Análisis Numérico

Fac. de Matemáticas, Univ. de Sevilla

C/. Tarfia s/n, 41012 - Sevilla, SPAIN,

E-mail addresses: jjgarmel@ull.es, jrossi@dm.uba.ar, suarez@us.es

\begin{abstract}
In this work we consider existence and uniqueness of positive solutions to the elliptic equation $-\Delta u=\lambda u$ in $\Omega$, with the nonlinear boundary conditions $\frac{\partial u}{\partial \nu}=u^{p}$ on $\Gamma_{1}, \frac{\partial u}{\partial \nu}=-u^{q}$ on $\Gamma_{2}$, where $\Omega$ is a smooth bounded domain, $\partial \Omega=\Gamma_{1} \cup \Gamma_{2}$, $\Gamma_{1} \cap \Gamma_{2}=\emptyset, \nu$ is the outward unit normal, $p, q>0$ and $\lambda$ is a real parameter. We obtain a complete picture of the bifurcation diagram of the problem, depending on the values of $p, q$ and the parameter $\lambda$. Our proofs are based on different techniques: variational arguments, bifurcation techniques or comparison arguments, depending on the range of parameters considered.
\end{abstract}

Keywords: Elliptic problems, nonlinear boundary conditions, sub and supersolutions, bifurcation.

AMS 2000 Subject Classification: 35J65, 35J25, 35B32.

\section{Introduction}

The aim of the present work is the study of existence and uniqueness (or multiplicity) of positive solutions to the following elliptic problem

$$
\left\{\begin{aligned}
-\Delta u & =\lambda u \quad \text { in } \quad \Omega, \\
\frac{\partial u}{\partial \nu} & =u^{p} \quad \text { on } \quad \Gamma_{1}, \\
\frac{\partial u}{\partial \nu} & =-u^{q} \quad \text { on } \quad \Gamma_{2} .
\end{aligned}\right.
$$

Here $\Omega$ is a $C^{2, \alpha}$ domain of $\mathbb{R}^{m}, \partial \Omega=\Gamma_{1} \cup \Gamma_{2}, \Gamma_{1} \cap \Gamma_{2}=\emptyset$ and $\nu$ is the outward unit normal vector field. The exponents $p, q$ are positive and $\lambda$ will be regarded as a real parameter without definite sign. 
Elliptic problems with non-linear boundary conditions have been deeply analyzed in recent years, see the survey [31] and references therein. Boundary conditions of the kind

$$
\frac{\partial u}{\partial \nu}= \pm u^{p}
$$

arise naturally in many situations. For instance when considering extremals for the Sobolev trace embedding ([31]) or when modelling chemical reactions due to the presence of a solid catalyzer, see [27]. Another motivation comes from geometry. One is lead to nonlinear boundary conditions when performing a description of conformal deformations on Riemannian manifolds with boundary, see [9], [13] and [14].

The main novelty in this paper is that in problem (1.1) both signs in (1.2) appear in different parts of $\partial \Omega$. Thus there exists a competition between an incoming flux at one part of the boundary, $\Gamma_{1}$ (given by the term $u^{p}$ ), and an outgoing flux on another part, $\Gamma_{2}$ (given by $-u^{q}$ ), while in the interior of the domain we only have a linear diffusion controlled with a parameter $\lambda$. It is interesting to look at the necessary balance between both fluxes and the linear diffusion to obtain existence and uniqueness (or multiplicity) of positive solutions to (1.1).

The only previous reference that deals with (1.1) is, at the best of our knowledge, the paper [15], where the authors describe the set of solutions to (1.1) for $\lambda=0$ in an interval, that is, solutions $w(x)$ to $w^{\prime \prime}(x)=0$ for $x \in(0, L)$ with the boundary conditions $-w^{\prime}(0)=w^{p}(0)$ and $w^{\prime}(L)=-w^{q}(L)$. They performed a complete description of the set of positive solutions to this problem in this particular case.

In this paper, we extend the above results and study problem (1.1) in general domains and for any $\lambda$. Remark that in the case of an interval with $\lambda=0$ solutions have the special form $w(x)=a x+b$. No such explicit form is available in the general case treated here, thus making the analysis much more involved.

We will perform a complete analysis of the bifurcation diagram of nonnegative solutions to (1.1) according to the values of $p, q$ and the parameter $\lambda$. To obtain our results we make use of different tools: variational arguments, bifurcation techniques or comparison arguments, according to the range of parameters considered. We remark that nonnegative nontrivial solutions to (1.1) are strictly positive in $\bar{\Omega}$, thanks to the maximum and Hopf's principles. According to standard elliptic theory, they are also classical.

Before proceeding to the statements of our theorems, we need to introduce some notation regarding principal eigenvalues. For $h \in C\left(\Gamma_{1}\right), g \in C\left(\Gamma_{2}\right)$, we denote by $\Lambda(N+h, N+g)$ the principal eigenvalue of the problem

$$
\left\{\begin{array}{r}
-\Delta \phi=\lambda \phi \quad \text { in } \quad \Omega, \\
\frac{\partial \phi}{\partial \nu}+h \phi=0 \quad \text { on } \quad \Gamma_{1} \\
\frac{\partial \phi}{\partial \nu}+g \phi=0 \quad \text { on } \quad \Gamma_{2}
\end{array}\right.
$$

and by $\Lambda(N+h, D)$ that of the problem

$$
\left\{\begin{array}{lll}
-\Delta \phi=\lambda \phi & \text { in } & \Omega \\
\frac{\partial \phi}{\partial \nu}+h \phi=0 & \text { on } & \Gamma_{1} \\
\phi=0 & \text { on } & \Gamma_{2}
\end{array}\right.
$$


We will quote some important properties of $\Lambda(N+h, N+g)$ and $\Lambda(N+h, D)$ in Section 2 .

We are now ready to state our results. For the sake of clarity, we divide the exposition in several cases according to the set of exponents $p$ and $q$. We find that very different pictures appear.

Case I. $p<1<q$.

Theorem 1.1. Assume that $p<1<q$. Then problem (1.1) does not have positive solutions if $\lambda \geq \Lambda(N, D)$, while it has a unique positive solution for $\lambda<\lambda(N, D)$.

Case II. $p, q>1$.

Theorem 1.2. Assume that $1<q$ and $1<p<m /(m-2)$. Then:

1. There exists $\Lambda, 0 \leq \Lambda \leq \Lambda(N, D)$ such that, for $\lambda>\Lambda$, problem (1.1) does not have positive solutions, while it has at least a positive solution if $\lambda<\Lambda$.

2. The solutions are bounded in $L^{\infty}$ for bounded values of $\lambda$.

3. If $\Lambda>0$, then there exists at least two positive solutions for $\lambda \in(0, \Lambda)$, and a positive solution for $\lambda=\Lambda$. In addition, there exists a minimal positive solution for every such $\lambda \in(0, \Lambda)$.

4. If $p>q$ or $p=q$ and $\left|\Gamma_{2}\right|>\left|\Gamma_{1}\right|$, then $\Lambda>0$, and the minimal solution is the unique stable positive solution to (1.1) for $\lambda \in(0, \Lambda)$.

5. If $p=q$ and $\left|\Gamma_{2}\right| \leq\left|\Gamma_{1}\right|$, then $\Lambda=0$.

Case III. $p, q<1$.

Theorem 1.3. Assume that $p, q<1$.

1. There exists $\Lambda, 0 \leq \Lambda \leq \Lambda(N, D)$ such that problem (1.1) admits a positive solution for $\lambda<\Lambda$, while no positive solutions exist for $\lambda>\Lambda$.

2. In case $\Lambda>0$, there also exists a positive solution for $\lambda=\Lambda$.

3. If $p<q$ or $p=q$ and $\left|\Gamma_{2}\right|<\left|\Gamma_{1}\right|$, then $\Lambda>0$, while $\Lambda=0$ for $p=q$ and $\left|\Gamma_{2}\right| \geq\left|\Gamma_{1}\right|$.

4. When $p \leq q$, the solution is unique for $\lambda \leq 0$.

Case IV. $q<1<p$.

Theorem 1.4. Assume $q<1<p<m /(m-2)$. If $\lambda \geq \Lambda(N, D)$, then problem (1.1) does not have positive solutions and for $\lambda<\lambda(N, D)$, there exists at least a positive solution.

Case V. $q=1>p, q=1<p, p=1>q, p=1<q$.

Theorem 1.5. It holds,

1. Assume $q=1>p$. If $\lambda \geq \Lambda(N, N+1)$, then problem (1.1) does not have positive solutions, while for $\lambda<\Lambda(N, N+1)$, there exists a unique positive solution. 
2. Assume $q=1<p$. If $\lambda \geq \Lambda(N, N+1)$, then problem (1.1) does not have positive solutions. If in addition $p<m /(m-2)$ there exists at least a positive solution for $\lambda<\Lambda(N, N+1)$.

3. Assume $p=1>q$. There exists at least a positive solution to (1.1) if, and only if, $\lambda \in(\Lambda(N-1, N), \Lambda(N-1, D))$.

4. Assume $p=1<q$. There exists at least a positive solution to (1.1) if, and only if, $\lambda \in(\Lambda(N-1, N), \Lambda(N-1, D))$. In this case, the solution is unique.

See Figures 1 and 2 where we have represented the bifurcation diagrams in all the cases.

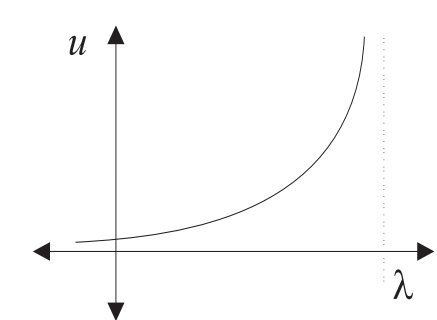

a)

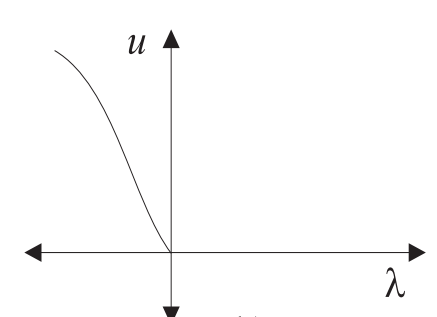

b)

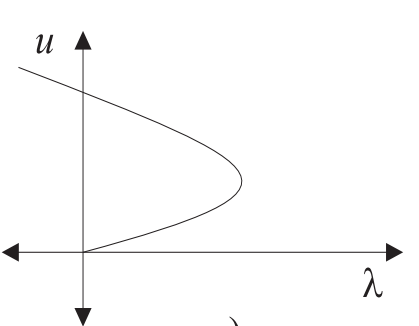

c)

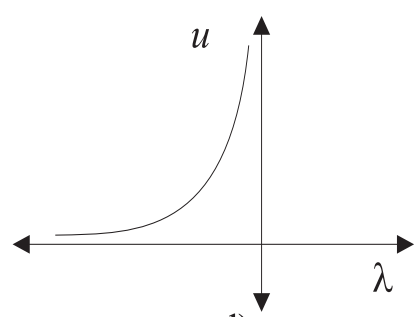

d)

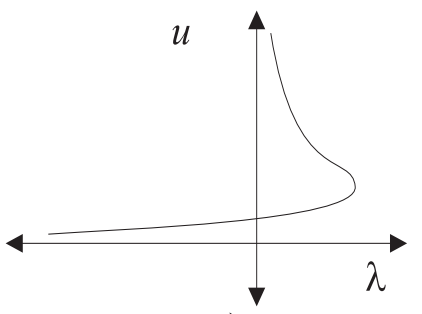

e)

Figure 1: Bifurcation diagrams of (1.1): Case a) $p<1<q$; Case b) $1<p<q$; Case c) $1<q<p<m /(m-2)$; Case d) $1>p>q$; Case e) $1>q>p$.

The case $p=q=1$ is not included in Theorem 1.5, since the problem becomes linear. In that case there is a single value of $\lambda$ for which a positive solution exists, namely the principal eigenvalue $\Lambda(N-1, N+1)$.

Let us briefly comment on some ideas and methods used in the proofs of these theorems. The classification in cases that we have chosen to present our results relies on the exponents $p$ and $q$ being super or sublinear. As we can observe from the figures, the picture changes completely when one of the exponents changes from being superlinear to sublinear. The limit cases (which we gather in Theorem 1.5) exhibit a sort of borderline behavior.

In the case $p<1<q$ the reaction is sublinear while the absorption is superlinear; this leads to the use of super and subsolutions to obtain existence of a unique positive solution (for $\lambda<\Lambda(N, D)$ ). When $p, q<1$ we use a bifurcation analysis at the point $(0, \infty)$, 


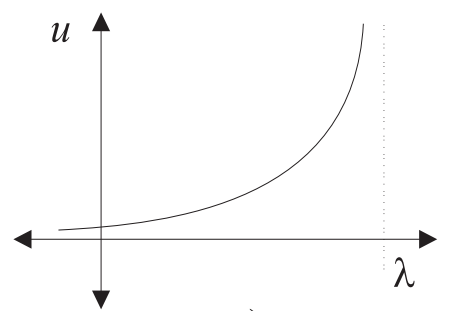

a)

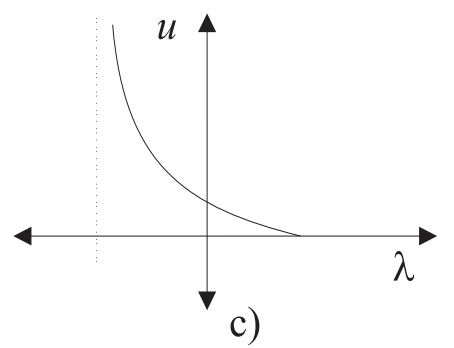

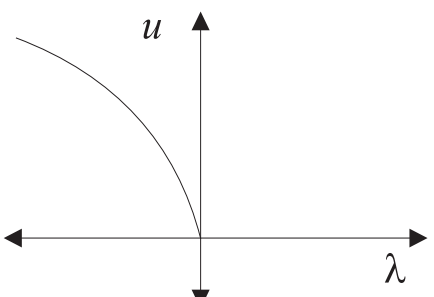

b)

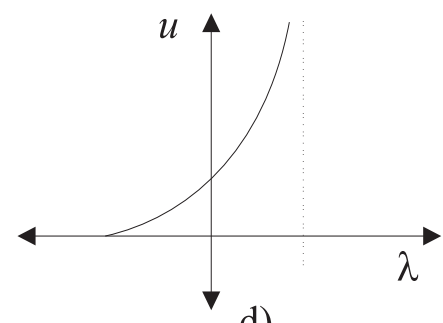

d)

Figure 2: Bifurcation diagrams of (1.1): Case a) $q=1>p$; Case b) $q=1<p<m /(m-2)$; Case c) $p=1>q$; Case d) $p=1<q$.

while for $p, q>1$ we have a bifurcation from $(0,0)$. The a priori bounds on solutions for subcritical $p, p<m /(m-2)$, which are needed in this case, come from the use of the well-known blow-up technique introduced in [19]. Observe that the exponent $p_{c}=$ $m /(m-2)$ is critical with respect to the Sobolev trace embedding $H^{1}(\Omega) \hookrightarrow L^{p+1}\left(\Gamma_{1}\right)$. The restriction on $p$ being subcritical also appears when variational arguments (the mountain pass theorem) are used. This will be done for the case $q<1<p<p_{c}$.

The rest of the paper is organized as follows: in Section 2, we present some general results on principal eigenvalues and uniqueness of positive solutions. Sections 3 and 4 are dedicated to obtain a priori bounds for the solutions and to some bifurcation results, respectively. Finally, Section 5 is devoted to the proof of the theorems; each theorem will be treated in a different subsection.

\section{Some preliminaries and general results}

In this section we present some known results which will be used along the paper, and we will analyze some basic features of problem (1.1).

We begin by recalling that, thanks to Hopf's maximum principle, nonnegative nontrivial weak solutions $u \in H^{1}(\Omega)$ to (1.1) are strictly positive in $\bar{\Omega}$, and hence, by standard regularity theory of elliptic equations (cf. [20] and [25]) $u \in C^{2, \alpha}(\bar{\Omega}) \cap C^{\infty}(\Omega)$, no matter the values of $p$ and $q$.

Now let us come back to the eigenvalue problem quoted in the introduction. For 
$h \in C\left(\Gamma_{1}\right), g \in C\left(\Gamma_{2}\right)$, consider

$$
\left\{\begin{array}{cc}
-\Delta \phi=\lambda \phi & \text { in } \Omega, \\
\frac{\partial \phi}{\partial \nu}+h \phi=0 & \text { on } \Gamma_{1}, \\
\frac{\partial \phi}{\partial \nu}+g \phi=0 & \text { on } \Gamma_{2} .
\end{array}\right.
$$

This problem has a unique principal eigenvalue, that is, a unique eigenvalue which has an associated positive eigenfunction. It will be denoted by $\Lambda(N+h, N+g)$. We are also using the notation $D$ for the Dirichlet boundary condition. That is, we are using the notation $\Lambda(N+h, D)$ for the first eigenvalue of the problem

$$
\begin{cases}-\Delta \phi=\lambda \phi & \text { in } \Omega, \\ \frac{\partial \phi}{\partial \nu}+h \phi=0 & \text { on } \Gamma_{1}, \\ \phi=0 & \text { on } \Gamma_{2},\end{cases}
$$

and symmetrically $\Lambda(D, N+g)$ when the Dirichlet condition is imposed on $\Gamma_{1}$. Finally, let $\Lambda(D, D)$ be the first eigenvalue when Dirichlet homogeneous conditions are imposed on the whole $\partial \Omega$.

Next we state some important properties of these principal eigenvalues, when varying the functions $h$ and $g$ and the domain $\Omega$. See [8] for very general results of this type.

Proposition 2.1. For fixed $h \in C\left(\Gamma_{1}\right)$, the mapping $g \in C\left(\Gamma_{2}\right) \mapsto \Lambda(N+h, N+g) \in \mathbb{R}$ is continuous and increasing. Moreover

$$
\begin{aligned}
& \lim _{K \rightarrow-\infty} \Lambda(N+h, N+K)=-\infty \\
& \lim _{K \rightarrow+\infty} \Lambda(N+h, N+K)=\Lambda(N+h, D) .
\end{aligned}
$$

If in addition $\Lambda_{\delta}(N+h, D)$ stands for the first eigenvalue of the problem

$$
\begin{cases}-\Delta \phi=\lambda \phi & \text { in } \Omega_{\delta} \\ \frac{\partial \phi}{\partial \nu}+h \phi=0 & \text { on } \Gamma_{1} \\ \phi=0 & \text { on } \Gamma_{2, \delta}\end{cases}
$$

where $\Omega_{\delta}=\Omega \cup\left\{x \in \mathbb{R}^{m}: \operatorname{dist}\left(x, \Gamma_{2}\right)<\delta\right\}$ and $\Gamma_{2, \delta}=\left\{x \in \mathbb{R}^{m} \backslash \Omega: \operatorname{dist}\left(x, \Gamma_{2}\right)=\delta\right\}$, then

$$
\lim _{\delta \rightarrow 0} \Lambda_{\delta}(N+h, D)=\Lambda(N+h, D) .
$$

Similar results are valid for the eigenvalue $\Lambda(N+h, N+g)$ when $g \in C\left(\Gamma_{2}\right)$ is kept fixed.

Remark 1. For the eigenvalue $\Lambda(N+h, D)$ we also have an asymptotic property analogous to $(2.2)$, namely

$$
\begin{aligned}
& \lim _{K \rightarrow-\infty} \Lambda(N+K, D)=-\infty \\
& \lim _{K \rightarrow+\infty} \Lambda(N+K, D)=\Lambda(D, D) .
\end{aligned}
$$


The rest of the section is devoted to obtain some preliminary results which are applicable to problem (1.1) in several ranges of the parameters. We show next that the eigenvalue $\Lambda(N, D)$ is an upper bound for the set of $\lambda$ 's for which there exists a positive solution. We remark that this nonexistence result is valid for all cases, although in some situations the upper limit can be improved.

Lemma 2.2. Let $\Lambda=\Lambda(N, D)$ be the first eigenvalue of (2.1) with $h=0$. If $\lambda \geq \Lambda$ then there is no positive solution to (1.1).

Proof. Assume by contradiction that there exists a positive solution $u$ of (1.1) with $\lambda \geq \Lambda$. Let $\phi$ be a positive eigenfunction associated to $\Lambda$. From the weak formulation of (1.1) using $\phi$ as test function we obtain

$$
\int_{\Omega} \nabla u \cdot \nabla \phi-\lambda \int_{\Omega} u \phi=\int_{\Gamma_{1}} u^{p} \phi
$$

Integrating by parts and using that $\phi$ is an eigenfunction (which verifies $\frac{\partial \phi}{\partial \nu}<0$ on $\Gamma_{2}$ ) we get

$$
0 \geq(\Lambda-\lambda) \int_{\Omega} u \phi=\int_{\Gamma_{1}} u^{p} \phi-\int_{\Gamma_{2}} u \frac{\partial \phi}{\partial \nu}>0
$$

a contradiction.

We now consider the issue of uniqueness of positive solutions to (1.1). We include a proof for completeness (see however [29] and [18]).

Lemma 2.3. Let $0 \leq p \leq 1$ and $q \geq 1$, with $p$ and $q$ not simultaneously equal to 1 . Then there exists at most one positive solution to (1.1).

Proof. We use ideas from [7]. Take two positive solutions $u_{1}$ and $u_{2}$, then

$$
\int_{\Omega}\left(\frac{-\Delta u_{1}}{u_{1}}+\frac{\Delta u_{2}}{u_{2}}\right)\left(u_{1}^{2}-u_{2}^{2}\right)=0 .
$$

On the other hand, integrating by parts, we obtain that

$$
\int_{\Omega}-\Delta u_{1}\left(\frac{u_{1}^{2}-u_{2}^{2}}{u_{1}}\right)=\int_{\Omega} \nabla u_{1} \cdot \nabla\left(\frac{u_{1}^{2}-u_{2}^{2}}{u_{1}}\right)-\int_{\Gamma_{1}} u_{1}^{p-1}\left(u_{1}^{2}-u_{2}^{2}\right)+\int_{\Gamma_{2}} u_{1}^{q-1}\left(u_{1}^{2}-u_{2}^{2}\right),
$$

and hence, using (2.4) we get

$$
\begin{aligned}
0=\int_{\Omega} & \nabla u_{1} \cdot \nabla\left(\frac{u_{1}^{2}-u_{2}^{2}}{u_{1}}\right)-\nabla u_{2} \cdot \nabla\left(\frac{u_{1}^{2}-u_{2}^{2}}{u_{2}}\right) \\
& +\int_{\Gamma_{1}}\left(u_{2}^{p-1}-u_{1}^{p-1}\right)\left(u_{1}^{2}-u_{2}^{2}\right)+\int_{\Gamma_{2}}\left(u_{1}^{q-1}-u_{2}^{q-1}\right)\left(u_{1}^{2}-u_{2}^{2}\right) .
\end{aligned}
$$

Finally, since

$$
\int_{\Omega} \nabla u_{1} \cdot \nabla\left(\frac{u_{1}^{2}-u_{2}^{2}}{u_{1}}\right)-\nabla u_{2} \cdot \nabla\left(\frac{u_{1}^{2}-u_{2}^{2}}{u_{2}}\right)=\int_{\Omega} u_{1}^{2}\left|\nabla\left(\frac{u_{2}}{u_{1}}\right)\right|^{2}+u_{2}^{2}\left|\nabla\left(\frac{u_{1}}{u_{2}}\right)\right|^{2},
$$

we obtain from (2.5) that

$$
\int_{\Gamma_{1}}\left(u_{2}^{p-1}-u_{1}^{p-1}\right)\left(u_{1}^{2}-u_{2}^{2}\right)+\int_{\Gamma_{2}}\left(u_{1}^{q-1}-u_{2}^{q-1}\right)\left(u_{1}^{2}-u_{2}^{2}\right) \leq 0 .
$$

Since $p \leq 1 \leq q$, not both equal to 1 , we conclude that $u_{1}=u_{2}$. 
Remark 2. The proof of Lemma 2.3 continues to be valid if we replace the nonlinear boundary condition on $\Gamma_{1}$ or $\Gamma_{2}$ (or both) by zero, or by a nonhomogeneous Dirichlet condition. This will be also used in the sequel.

We now include a further uniqueness result in a situation where Lemma 2.3 is not applicable.

Lemma 2.4. If $1>q \geq p$ and $\lambda \leq 0$ then there exists at most one positive solution to (1.1).

Proof. Let $u_{1}, u_{2}$ be positive solutions to (1.1). We want to show that $u_{1}=u_{2}$. Let

$$
v_{1}(x)=u_{1}^{1-q}(x) \quad \text { and } \quad v_{2}(x)=u_{2}^{1-q}(x) .
$$

It is not hard to show that

$$
-\Delta v_{i}=\frac{q}{1-q} \frac{\left|\nabla v_{i}\right|^{2}}{v_{i}}+\lambda(1-q) v_{i}
$$

for $i=1,2$. Now let $w(x)=v_{1}(x)-v_{2}(x)$ and assume that $w$ has a positive maximum in $\bar{\Omega}$. We have

$$
\begin{cases}-\Delta w-\frac{q}{1-q} \frac{1}{v_{1}} \nabla w \cdot \nabla\left(v_{1}+v_{2}\right)+\frac{q}{1-q}\left(\frac{\left|\nabla v_{2}\right|^{2}}{v_{1} v_{2}}\right) w-\lambda(1-q) w=0 & \text { in } \Omega \\ \frac{\partial w}{\partial \nu}=(1-q)\left(v_{1}^{\frac{p-q}{1-q}}-v_{2}^{\frac{p-q}{1-q}}\right) & \text { on } \Gamma_{1} \\ \frac{\partial w}{\partial \nu}=0 & \text { on } \Gamma_{2} .\end{cases}
$$

Since the coefficient of $w$ in the equation is positive, the strong maximum principle and Hopf's principle imply that $w$ is a positive constant $c$. However, this is impossible, since the unique constant which solves the previous equation is $c=0$.

We conclude that $w$ does not have a positive maximum in $\Omega$, and thus $v_{1} \leq v_{2}$. By a symmetric argument we get $v_{1}=v_{2}$, and the uniqueness of the positive solution is proved.

\section{A priori bounds}

This section is dedicated to prove that we have a priori bounds for the positive solutions to (1.1) in the cases $q \geq 1,1<p<m /(m-2)$. We consider first the case $q>1$. For our argument below, we need to prove existence and uniqueness of solutions to an auxiliary problem.

Lemma 3.1. Let $q>1$ and $R>0$ and consider the problem:

$$
\begin{cases}-\Delta z=\mu z & \text { in } \Omega, \\ z=R & \text { on } \Gamma_{1}, \\ \frac{\partial z}{\partial \nu}=-z^{q} & \text { on } \Gamma_{2} .\end{cases}
$$

Then, there exists a positive solution to (3.1) if, and only if, $\mu<\Lambda(D, D)$. Moreover, the solution is unique. 
Proof. Uniqueness follows by Lemma 2.3 (see Remark 2). Assume that there exists a positive solution $z$ of $(3.1)$. Let $\phi$ be the eigenfunction associated to $\Lambda(D, D)$. Multiplying (3.1) by $\phi$ and integrating by parts, we get

$$
\int_{\partial \Omega} \frac{\partial \phi}{\partial \nu} z=(\mu-\Lambda(D, D)) \int_{\Omega} z \phi
$$

whence we deduce that $\mu<\Lambda(D, D)$ since $\partial \phi / \partial \nu<0$ on $\partial \Omega$.

To prove existence, we will use the method of sub and supersolutions. It is clear that $\underline{z}=0$ is subsolution of (3.1). We are next constructing a supersolution. Take $\mu<\Lambda(D, D)$. By (2.2) in Proposition 2.1, there exists $\rho>0$ (large enough) such that $\mu<\Lambda(D, N+\rho)$. Thanks to (2.3) in Proposition 2.1, we can find a small $\delta$ such that $\mu<\Lambda_{\delta}(D, N+\rho)$, where $\Lambda_{\delta}(D, N+\rho)$ denotes the first eigenvalue of the problem

$$
\begin{cases}-\Delta \phi=\lambda \phi & \text { in } \Omega_{\delta}, \\ \phi=0 & \text { on } \Gamma_{1, \delta} \\ \frac{\partial \phi}{\partial \nu}+\rho \phi=0 & \text { on } \Gamma_{2},\end{cases}
$$

with $\Omega_{\delta}=\Omega \cup\left\{x \in \mathbb{R}^{m}: \operatorname{dist}\left(x, \Gamma_{1}\right)<\delta\right\}$ and $\Gamma_{1, \delta}=\left\{x \in \mathbb{R}^{m} \backslash \Omega\right.$ : $\left.\operatorname{dist}\left(x, \Gamma_{1}\right)=\delta\right\}$. Let $\phi_{1}$ be the eigenfunction associated to this eigenvalue (which is strictly positive in $\bar{\Omega}$ ). It can be proved that $\bar{z}=K \phi_{1}$ is supersolution of (3.1). Indeed, in $\Omega$

$$
-\Delta \bar{z}=K \Lambda_{\delta}(D, N+\rho) \phi_{1}>\mu \bar{z},
$$

while on $\Gamma_{1}$

$$
\bar{z}=K \phi_{1}>R
$$

for large $K$, and finally on $\Gamma_{2}$

$$
\frac{\partial \bar{z}}{\partial \nu}=-K \rho \phi_{1} \geq-K^{q} \phi_{1}^{q}
$$

which also holds for sufficiently large $K$. Since $\underline{z} \leq \bar{z}$, the existence of a solution follows.

In order to simplify the proof of the a priori bounds below, we are showing next that it is sufficient to get a bound for the solutions on $\Gamma_{1}$.

Lemma 3.2. Let $I \subset(-\infty, \Lambda(N, D))$ be a bounded interval, and assume that there exists a positive constant $M$ such that

$$
\sup _{\Gamma_{1}} u \leq M
$$

for every positive solution $(\lambda, u)$ to (1.1) with $\lambda \in I$. Then, there exists a positive constant C such that

$$
\sup _{\bar{\Omega}} u \leq C
$$

for every positive solution $(\lambda, u)$ to (1.1) with $\lambda \in I$.

Proof. First of all notice that $\Lambda(N, D)<\Lambda(D, D)$. Thus there exists a unique positive solution to (3.1) with $\mu=\Lambda(N, D)$ and $R=M$, which we are denoting by $z$.

On the other hand, if $(\lambda, u)$ is a solution to (1.1) with $\lambda \in I$, then $\lambda<\Lambda(N, D)$, and it follows that $u$ is a subsolution to (3.1) with $\mu=\Lambda(N, D)$ and $R=M$. Since there exist arbitrarily large supersolutions (cf. the proof of Lemma 3.1), we deduce thanks to uniqueness that $u \leq z$. This concludes the proof with $C=\sup _{\bar{\Omega}} z$. 
Finally, we prove the a priori bounds. We use a blow-up argument as in [19].

Lemma 3.3. If $q>1,1<p<m /(m-2)$ and $I \subset(-\infty, \Lambda(N, D))$ is a bounded interval, then there exists a constant $C$ such that

$$
\sup _{\bar{\Omega}} u \leq C
$$

for every positive solution $(\lambda, u)$ with $\lambda \in I$.

Proof. By Lemma 3.2 it is enough to show that the solutions are bounded on $\Gamma_{1}$. We argue by contradiction: assume that the conclusion is false and hence there exists a sequence $\left\{\lambda_{n}\right\} \subset I$ with corresponding solutions $u_{n}$, such that

$$
M_{n}=\max _{\Gamma_{1}} u_{n}=u_{n}\left(x_{n}\right) \rightarrow+\infty .
$$

With no loss of generality, we may assume $\lambda_{n} \rightarrow \lambda_{0}$ and $x_{n} \rightarrow x_{0} \in \Gamma_{1}$. Let

$$
v_{n}(y)=\frac{u_{n}\left(x_{n}+M_{n}^{-(p-1)} y\right)}{M_{n}} .
$$

This sequence is uniformly bounded and verifies $v_{n}(0)=\max _{\bar{\Omega}} v_{n}(x)=1$ and

$$
\left\{\begin{aligned}
-\Delta v_{n} & =\frac{\lambda_{n}}{M_{n}^{2(p-1)}} v_{n} & & \text { in } \Omega_{n}, \\
\frac{\partial v_{n}}{\partial \nu} & =v_{n}^{p} & & \text { on }\left(\Gamma_{1}\right)_{n} \\
\frac{\partial v_{n}}{\partial \nu} & =-M_{n}^{q-p} v_{n}^{q} & & \text { on }\left(\Gamma_{2}\right)_{n} .
\end{aligned}\right.
$$

Where $\Omega_{n}=\left\{y: x_{n}+M_{n}^{-(p-1)} y \in \Omega\right\}, \Gamma_{i, n}=\left\{y: x_{n}+M_{n}^{-(p-1)} y \in \Gamma_{i}\right\}, i=1,2$ (observe that $\Omega_{n} \rightarrow \mathbb{R}_{+}^{m}, \Gamma_{1, n} \rightarrow \partial \mathbb{R}_{+}^{m}$ ). It is now a standard matter to pass to the limit (see the details in [17]) and obtain that $v_{n} \rightarrow v$, which is a positive and bounded solution to

$$
\left\{\begin{aligned}
-\Delta v & =0 & & \text { in } \mathbb{R}_{+}^{m}, \\
\frac{\partial v}{\partial \nu} & =v^{p} & & \text { on } \partial \mathbb{R}_{+}^{m},
\end{aligned}\right.
$$

with $v(0)=1$, a contradiction with the nonexistence result of [22], since $p$ is subcritical, $1<p<m /(m-2)$.

We finally consider the case $q=1,1<p<m /(m-2)$. Since the arguments are entirely similar to those used before, we only sketch the proof.

Lemma 3.4. If $q=1,1<p<m /(m-2)$ and $I \subset(-\infty, \Lambda(N, N+1))$ is a bounded interval, then there exists a constant $C$ such that

$$
\sup _{\bar{\Omega}} u \leq C,
$$

for every positive solution $(\lambda, u)$ with $\lambda \in I$. 
Sketch of the proof. It follows exactly as in Lemma 3.1 that the problem

$$
\begin{cases}-\Delta z=\mu z & \text { in } \Omega, \\ z=R & \text { on } \Gamma_{1}, \\ \frac{\partial z}{\partial \nu}+z=0 & \text { on } \Gamma_{2},\end{cases}
$$

where $R>0$, has a unique solution if and only if $\mu<\Lambda(D, N+1)$. Thus with an argument as in Lemma 3.2 (considering the solution to (3.2) with $\mu=\Lambda(N, N+1)<\Lambda(D, N+1)$ ) it follows that we only need to bound the solutions on $\Gamma_{1}$. It is easily seen that, once this is done, the proof of Lemma 3.3 carries over to the present situation with no changes at all, since the exponent $q$ did not play any role there.

\section{Bifurcation results}

This section is dedicated to some results on bifurcation from infinity and from zero. The first statement is related with bifurcation from infinity and it is well known when one of the two parts of the boundary, $\Gamma_{1}$ or $\Gamma_{2}$, is not present, see [5] and [32].

Proposition 4.1. Assume $q<1$ and $p \leq 1$. There exists an unbounded continuum $\mathcal{C}_{\infty} \subset \mathbb{R} \times C(\bar{\Omega})$ of positive solutions to (1.1) bifurcating from infinity at $\lambda=\lambda_{1}$, with $\lambda_{1}=\Lambda(N-1, N)$ when $p=1$ and $\lambda_{1}=0$ when $p<1$. Moreover, this is the unique bifurcation point from infinity. Furthermore, if $\delta_{0}>0$ is small enough and $\mathcal{N}=\left[\lambda_{1}-\right.$ $\left.\delta_{0}, \lambda_{1}+\delta_{0}\right] \times\left\{u \in C(\bar{\Omega}):\|u\|_{\infty} \geq 1\right\}$,

1. either $\mathcal{C}_{\infty} \backslash \mathcal{N}$ is bounded in $\mathbb{R} \times C(\bar{\Omega})$. In this case, $\mathcal{C}_{\infty} \backslash \mathcal{N}$ meets the set $\{(\lambda, 0)$ : $\lambda \in \mathbb{R}\}$, or

2. $\mathcal{C}_{\infty} \backslash \mathcal{N}$ is unbounded in $\mathbb{R} \times C(\bar{\Omega})$.

Finally, if $p>q$ the bifurcation direction is to the left, if $p<q$ the bifurcation is to the right and if $p=q$ it is to the right when $\left|\Gamma_{2}\right|>\left|\Gamma_{1}\right|$ or to the left when $\left|\Gamma_{2}\right|<\left|\Gamma_{1}\right|$.

Proof. The existence of the continuum of positive solutions to (1.1) bifurcating from infinity follows analogously to [5]. Let us show the assertion on the bifurcation direction. Assume that $p, q<1$ and let $\left(\lambda_{n}, u_{n}\right)$ be positive solutions to (1.1) with $\lambda_{n} \rightarrow 0$ and $\left\|u_{n}\right\|_{\infty} \rightarrow \infty$ as $n \rightarrow \infty$. Integrating (1.1) we get

$$
-\int_{\Gamma_{1}} u_{n}^{p}+\int_{\Gamma_{2}} u_{n}^{q}=\lambda_{n} \int_{\Omega} u_{n}
$$

Dividing by $\left\|u_{n}\right\|_{\infty}^{p}$, we have

$$
-\int_{\Gamma_{1}}\left(\frac{u_{n}}{\left\|u_{n}\right\|_{\infty}}\right)^{p}+\left\|u_{n}\right\|_{\infty}^{q-p} \int_{\Gamma_{2}}\left(\frac{u_{n}}{\left\|u_{n}\right\|_{\infty}}\right)^{q}=\lambda_{n}\left\|u_{n}\right\|^{1-p} \int_{\Omega}\left(\frac{u_{n}}{\left\|u_{n}\right\|_{\infty}}\right) .
$$

Taking into account that $u_{n} /\left\|u_{n}\right\|_{\infty} \rightarrow 1$ in $C(\bar{\Omega})$ (cf. [5]), we have that $\lambda_{n}<0$ when $p>q$, while $\lambda_{n}>0$ when $p<q$. Finally, if $p=q$, the left-hand side of (4.1) converges to $-\left|\Gamma_{1}\right|+\left|\Gamma_{2}\right|$, and this implies that for large $n, \operatorname{sgn} \lambda_{n}=\operatorname{sgn}\left(\left|\Gamma_{2}\right|-\left|\Gamma_{1}\right|\right)$. The proof for $p=1$ is similar. 
The following proposition is related to bifurcation from the trivial solution, $u \equiv 0$, see [5] and [28].

Proposition 4.2. Assume $p>1$ and $q \geq 1$. There exists an unbounded continuum $\mathcal{C}_{0} \subset \mathbb{R} \times C(\bar{\Omega})$ of positive solutions to (1.1) emanating from the trivial solution at $\lambda=$ $\lambda_{1}(N, N+1)$ when $q=1$ and $\lambda=0$ when $q>1$. Moreover, this is the unique point of bifurcation from the trivial solution, and with respect to the bifurcation direction:

1. If $p>q$ (resp. $p<q$ ) then the bifurcation direction is to the right (resp. to the left).

2. If $p=q$ then the bifurcation direction is to the right (resp. to the left) for $\left|\Gamma_{2}\right|>\left|\Gamma_{1}\right|$ (resp. $\left.\left|\Gamma_{2}\right|<\left|\Gamma_{1}\right|\right)$.

Proof. The existence of $\mathcal{C}_{0}$ follows by [28], and the bifurcation directions are calculated as in the proof of Proposition 4.1.

\section{$5 \quad$ Proofs of the theorems}

This final section is devoted to the proof of the theorems. We are dedicating a separate subsection to each theorem.

\subsection{Case I. $p<1<q$}

We analyze now the existence and uniqueness of positive solutions to (1.1) when the exponents verify $p<1<q$.

Proof of Theorem 1.1. Since uniqueness follows from Lemma 2.3, and nonexistence for $\lambda \geq \Lambda(N, D)$ from Lemma 2.2 , we only have to show existence for $\lambda<\Lambda(N, D)$. For this aim, we use the method of sub and supersolutions.

Let us look for the supersolution first. Since $\lambda<\Lambda(N, D)$, we have $\lambda<\Lambda(N-\eta, D)$ for small positive $\eta$, and thus Proposition 2.1 implies the existence of a unique $\theta$ such that $\lambda=\Lambda(N-\eta, N+\theta)$. Let $\phi$ be an associated normalized positive eigenfunction. That is, $\phi$ verifies

$$
\left\{\begin{aligned}
-\Delta \phi & =\lambda \phi & & \text { in } \Omega, \\
\frac{\partial \phi}{\partial \nu} & =\eta \phi & & \text { on } \Gamma_{1} \\
\frac{\partial \phi}{\partial \nu} & =-\theta \phi & & \text { on } \Gamma_{2} .
\end{aligned}\right.
$$

Our supersolution will be given by $\bar{u}=M \phi$. Indeed, the equation is trivially satisfied, while on $\Gamma_{1}$ we get

$$
\frac{\partial \bar{u}}{\partial \nu}=M \frac{\partial \phi}{\partial \nu}=M \eta \phi \geq M^{p} \phi^{p}=\bar{u}^{p}
$$

if $M$ is large enough. On $\Gamma_{2}$ we have

$$
\frac{\partial \bar{u}}{\partial \nu}=M \frac{\partial \phi}{\partial \nu}=-M \theta \phi \geq-M^{q} \phi^{q}=-\bar{u}^{q}
$$

taking $M$ larger if necessary. Therefore $\bar{u}$ is a supersolution to (1.1). 
To obtain a subsolution, since $\lambda<\Lambda(N, D)$ by Proposition 2.1 there exists $\eta>0$ such that $\lambda=\Lambda(N-\eta, D)$. Let $z$ a positive solution to

$$
\left\{\begin{array}{lll}
-\Delta z=\lambda z & \text { in } & \Omega \\
\frac{\partial z}{\partial \nu}=\eta z & \text { on } & \Gamma_{1} \\
z=0 & \text { on } & \Gamma_{2} .
\end{array}\right.
$$

Take

$$
\underline{u}=\varepsilon z
$$

as the desired subsolution. In $\Omega$ we have $-\Delta \underline{u}=\lambda \underline{u}$. On $\Gamma_{1}$ we get

$$
\frac{\partial \underline{u}}{\partial \nu}=\varepsilon \frac{\partial z}{\partial \nu}=\varepsilon \eta z \leq \varepsilon^{p} z^{p}=\underline{u}^{p}
$$

if $\varepsilon$ is small enough. On $\Gamma_{2}$ we just have $\underline{u}=0$. Therefore $\underline{u}$ is a subsolution to (1.1).

By taking a small enough $\varepsilon$ or a large enough $M$, we can achieve $\underline{u} \leq \bar{u}$, and the existence of a positive solution follows. This concludes the proof.

\subsection{Case II. $p, q>1$}

In this section we deal with the case $p, q>1$. Since the proof of Theorem 1.2 is the most complicated one, we divide it in several lemmas.

Lemma 5.1. There exists $\Lambda, 0 \leq \Lambda \leq \Lambda(N, D)$ such that, for $\lambda>\Lambda$ problem (1.1) does not have positive solutions, while it has a positive solution for $\lambda<\Lambda$.

Proof. We first remark that the nonexistence of positive solutions for $\lambda \geq \Lambda(N, D)$ follows by Lemma 2.2. Thus, let

$$
\Lambda=\sup \{\lambda \in \mathbb{R}:(1.1) \text { has at least a positive solution }\},
$$

which verifies $\Lambda \leq \Lambda(N, D)<\infty$. Moreover, $\Lambda \geq 0$, since, according to Proposition 4.2, an unbounded continuum $\mathcal{C}_{0}$ of positive solutions to (1.1) bifurcates from $(0,0)$. Since in addition we have bounds for the solutions thanks to Lemma 3.3, we deduce that there exists a positive solution for every $\lambda<0$. Thus only the existence of solutions when $\lambda \in(0, \Lambda)$ remains to be proved, provided that $\Lambda>0$.

Fix $\lambda \in(0, \Lambda)$, so there exists $\lambda_{0} \in(\lambda, \Lambda)$ such that for $\lambda=\lambda_{0}$ there exists a positive solution to (1.1), denoted by $u_{0}$. It is clear that $u_{0}$ is supersolution of (1.1) for $\lambda \leq \lambda_{0}$. For this $\lambda$ there exists $\theta>0$ for which $\lambda=\Lambda(N, N+\theta)$. Let $\phi$ be an associated positive eigenfunction, that is, a solution to

$$
\left\{\begin{aligned}
-\Delta \phi & =\lambda \phi & & \text { in } \Omega \\
\frac{\partial \phi}{\partial \nu} & =0 & & \text { on } \Gamma_{1} \\
\frac{\partial \phi}{\partial \nu} & =-\theta \phi & & \text { on } \Gamma_{2}
\end{aligned}\right.
$$

It is easy to show that $\underline{u}=\varepsilon \phi$ is subsolution of (1.1) for small $\varepsilon$. Hence, there exists a positive solution to (1.1) for every $\lambda \in(0, \Lambda)$. This concludes the proof. 
We are next showing that, in case $\Lambda>0$, a minimal positive solution exists for every $\lambda \in(0, \Lambda)$.

Lemma 5.2. Assume $\Lambda>0$. For every $0<\lambda<\Lambda$ there exists a minimal positive solution to $(1.1)$, denoted by $u_{\lambda}$.

Proof. Let us consider the problem

$$
\left\{\begin{aligned}
-\Delta w & =\lambda w & & \text { in } \Omega \\
\frac{\partial w}{\partial \nu} & =0 & & \text { on } \Gamma_{1} \\
\frac{\partial w}{\partial \nu} & =-w^{q} & & \text { on } \Gamma_{2} .
\end{aligned}\right.
$$

It can be proved as in Lemma 3.1 that problem (5.2) admits a unique positive solution if and only if $\lambda \in(0, \Lambda(N, D))$ (cf. Remark 2 for the uniqueness). We denote it by $w_{\lambda}$.

Moreover, every solution $u$ to (1.1) is a supersolution of (5.2), while we can construct subsolutions as small as desired, as in the proof of Lemma 5.1. Thus by uniqueness we have that $u(x) \geq w_{\lambda}(x)$, for every solution $u$ to (1.1). A standard iterative argument shows that the minimal solution $u_{\lambda}$ exists. This concludes the proof.

To prove the existence of two solutions and the uniqueness of the positive stable solution in $(0, \Lambda)$, we are going to use general results from [2]. Hence we need to show that our problem is in the general setting of [2].

Let $P$ be the cone of positive functions of $C(\bar{\Omega})$. With the ordering induced by $P$, $C(\bar{\Omega})$ is an ordered Banach space, with a normal cone which has nonempty interior, see Example 1.11 in [2]. Consider the interval $I=[-1, \Lambda+1]$ and let

$$
\beta>\sup _{\lambda \in I}\left\{\|u(\lambda)\|_{\infty}\right\}
$$

where $u(\lambda)$ is any solution to (1.1). Observe that since $p<m /(m-2)$, we have that $\beta<\infty$ (cf. Lemma 3.3). Take $K>0$, and rewrite our problem as

$$
\begin{cases}(-\Delta+K) u=(\lambda+K) u & \text { in } \Omega, \\ \frac{\partial u}{\partial \nu}=u^{p} & \text { on } \Gamma_{1}, \\ \frac{\partial u}{\partial \nu}+K u=K u-u^{q} & \text { on } \Gamma_{2} .\end{cases}
$$

Let $\mathcal{K}_{1}: C^{\alpha}(\bar{\Omega}) \mapsto C^{2, \alpha}(\bar{\Omega}), \alpha \in(0,1)$, be the operator such that $f \mapsto u=\mathcal{K}_{1} f$ being $u$ the solution to

$$
\left\{\begin{array}{cl}
(-\Delta+K) u=f & \text { in } \Omega, \\
\frac{\partial u}{\partial \nu}=0 & \text { on } \partial \Omega .
\end{array}\right.
$$

This operator can be extended to a linear compact and strongly positive map, denoted again by $\mathcal{K}_{1}, \mathcal{K}_{1}: C(\bar{\Omega}) \mapsto C^{1}(\bar{\Omega})$, see Theorem 4.2 in [2]. 
Consider now the operators $\mathcal{K}_{2}: C^{1, \alpha}\left(\Gamma_{1}\right) \mapsto C^{2, \alpha}(\bar{\Omega}), g \mapsto v=\mathcal{K}_{2} g$, where $v$ is the unique solution to

$$
\left\{\begin{array}{cc}
(-\Delta+K) v=0 & \text { in } \Omega, \\
\frac{\partial v}{\partial \nu}=g & \text { on } \Gamma_{1} \\
\frac{\partial v}{\partial \nu}=0 & \text { on } \Gamma_{2},
\end{array}\right.
$$

and $\mathcal{K}_{3}: C^{1, \alpha}\left(\Gamma_{2}\right) \mapsto C^{2, \alpha}(\bar{\Omega}), g \mapsto w=\mathcal{K}_{3} g$, where $w$ is the unique solution to

$$
\left\{\begin{array}{cc}
(-\Delta+K) w=0 & \text { in } \Omega \\
\frac{\partial w}{\partial \nu}=0 & \text { on } \Gamma_{1} \\
\frac{\partial w}{\partial \nu}+K w=g & \text { on } \Gamma_{2} .
\end{array}\right.
$$

Now, by [1], $\mathcal{K}_{2}$ and $\mathcal{K}_{3}$ can be extended to linear compact maps from $C(\partial \Omega)$ to $C(\bar{\Omega})$. Hence, it is not hard to prove that $u$ is a solution to (1.1) if, and only if,

$$
u=F(u, \lambda)=\mathcal{K}_{1}((\lambda+K) u)+\mathcal{K}_{2}\left(\gamma\left(u^{p}\right)\right)+\mathcal{K}_{3}\left(\gamma\left(K u-u^{q}\right)\right),
$$

where $\gamma: C(\bar{\Omega}) \mapsto C(\partial \Omega)$ is the trace operator.

On the other hand, $F$ is a differentiable operator. It is compact on bounded sets and strongly increasing for fixed $\lambda$ (cf. [2] for definitions). In addition, the partial derivatives

$$
\partial_{u} F\left(u_{0}, \lambda_{0}\right) \xi=\mathcal{K}_{1}\left(\lambda_{0}+K\right) \xi+\mathcal{K}_{2}\left(p \gamma\left(u_{0}^{p-1}\right)\right) \xi+\mathcal{K}_{3}\left(\gamma\left(K-q u_{0}^{q-1}\right)\right) \xi
$$

and

$$
\partial_{\lambda} F\left(u_{0}, \lambda_{0}\right) \mu=\mu \mathcal{K}_{1} u_{0}
$$

are also strongly positive provided $K$ is large enough. Indeed, $\mathcal{K}_{1}$ is strongly positive, and if $\xi$ is nonnegative and nontrivial, then $\eta=\partial_{u} F\left(u_{0}, \lambda_{0}\right) \xi$ is equivalent to $\eta=\eta_{1}+\eta_{2}$ where

$$
\eta_{1}=\mathcal{K}_{1}\left(\left(\lambda_{0}+K\right) \xi\right), \quad \eta_{2}=\mathcal{K}_{2}\left(p \gamma\left(u_{0}^{p-1}\right)\right) \xi+\mathcal{K}_{3}\left(\gamma\left(K-q u_{0}^{q-1}\right)\right) \xi .
$$

Taking $K>\max \left\{0,-\lambda_{0}\right\}$ it follows that $\eta_{1} \in \operatorname{int} P$. On the other hand, $\eta_{2}$ verifies

$$
\left\{\begin{array}{cl}
(-\Delta+K) \eta_{2}=0 & \text { in } \Omega, \\
\frac{\partial \eta_{2}}{\partial \nu}=p u_{0}^{p-1} \xi & \text { on } \Gamma_{1} \\
\frac{\partial \eta_{2}}{\partial \nu}+K \eta_{2}=\left(K-q u_{0}^{q-1}\right) \xi & \text { on } \Gamma_{2} .
\end{array}\right.
$$

So, if we take

$$
K>\max \left\{0,-\lambda_{0}, q \beta^{q-1}\right\},
$$

we get $K \geq q \beta^{q-1} \geq q u_{0}^{q-1}$, and so $\eta_{2} \in \operatorname{int} P$. This proves the claim, and shows that problem (1.1) can be cast into the general setting of [2]. We state now the already claimed existence result.

Lemma 5.3. Assume that $\Lambda>0$. Then, for all $\lambda \in(0, \Lambda)$ there exist at least two positive solutions to (1.1) and one positive solution for $\lambda=\Lambda$. 
Proof. We can directly apply Theorem 20.9 of [2] (see the arguments after Proposition 20.8 there and Theorem 7.4 in [3]) and conclude the existence of at least two positive solutions for $\lambda \in(0, \Lambda)$ and at least a positive solution for $\lambda=\Lambda$.

We remark that, if we denote $\rho=r\left(u_{0}, \lambda_{0}\right)$ the spectral radius of $\partial_{u} F\left(u_{0}, \lambda_{0}\right)$, then $\rho$ satisfies

$$
\frac{1}{\rho}\left(K+\lambda_{0}\right)-K=\Lambda\left(N-\frac{p}{\rho} u_{0}^{p-1}, N+K+\frac{1}{\rho}\left(q u_{0}^{q-1}-K\right)\right) .
$$

This will be used in what follows.

We finally proceed to show the uniqueness of the stable solution. We recall that given a positive solution $\left(\lambda_{0}, u_{0}\right)$ of (1.1) we say that it is stable (resp. unstable) if the principal eigenvalue of the linearization around $\left(\lambda_{0}, u_{0}\right)$ is positive (resp. negative), that is,

$$
\Lambda\left(N-p u_{0}^{p-1}, N+q u_{0}^{q-1}\right)>\lambda_{0} \quad\left(\operatorname{resp} . \Lambda\left(N-p u_{0}^{p-1}, N+q u_{0}^{q-1}\right)<\lambda_{0}\right) .
$$

We are then involved in proving the next result.

Theorem 5.4. Assume that $q<p$ or $q=p$ and $\left|\Gamma_{2}\right|>\left|\Gamma_{1}\right|$. Then the minimal positive solution $u_{\lambda}$ is the unique positive stable solution to (1.1) for all $\lambda \in(0, \Lambda)$.

The proof of Theorem 5.4 uses some important tools from [2] and [10]. The first result needed provides us with a complete picture of the structure of the set of positive solutions around a stable or neutrally stable solution (that is, the first eigenvalue of the linearization equals zero).

Lemma 5.5. Let $\left(\lambda_{0}, u_{0}\right)$ be a positive solution to (1.1) with $\lambda=\lambda_{0}$.

1. If $\left(\lambda_{0}, u_{0}\right)$ is stable, that is, if first inequality of (5.5) holds, then, there exists $\varepsilon>0$ and a differentiable mapping $u: I=\left(\lambda_{0}-\varepsilon, \lambda_{0}+\varepsilon\right) \mapsto P$ such that $u\left(\lambda_{0}\right)=u_{0}$ and $(\lambda, u(\lambda))$ is a positive solution to (1.1) for each $\lambda \in I$. Moreover, the mapping $\lambda \mapsto u(\lambda)$ is increasing and there exists a neighborhood $\mathcal{V}$ of $\left(\lambda_{0}, u_{0}\right)$ in $\mathbb{R} \times P$ such that if $(\lambda, u) \in \mathcal{V}$ is a solution to (1.1), then $(\lambda, u)=(\lambda, u(\lambda))$ for some $\lambda \in I$.

2. If $\left(\lambda_{0}, u_{0}\right)$ is neutrally stable, that is,

$$
\Lambda\left(N-p u_{0}^{p-1}, N+q u_{0}^{q-1}\right)=\lambda_{0},
$$

let $\Phi_{0}$ be the principal eigenfunction associated with $\Lambda\left(N-p u_{0}^{p-1}, N+q u_{0}^{q-1}\right)$, that is, $\Phi_{0}$ verifies

$$
\left\{\begin{array}{cc}
-\Delta \Phi_{0}-\lambda_{0} \Phi_{0}=0 & \text { in } \Omega, \\
\frac{\partial \Phi_{0}}{\partial \nu}-p u_{0}^{p-1} \Phi_{0}=0 & \text { on } \Gamma_{1}, \\
\frac{\partial \Phi_{0}}{\partial \nu}+q u_{0}^{q-1} \Phi_{0}=0 & \text { on } \Gamma_{2} .
\end{array}\right.
$$

Then, there exists $\varepsilon>0$ and a differentiable mapping $(\lambda, u): J=(-\varepsilon, \varepsilon) \mapsto \mathbb{R} \times P$ such that $(\lambda(0), u(0))=\left(\lambda_{0}, u_{0}\right)$ and for each $s \in J,(\lambda(s), u(s))$ is a positive solution to (1.1). Moreover,

$$
\lambda(s)=\lambda_{0}+s^{2} \lambda_{2}+O\left(s^{3}\right), \quad u(s)=u_{0}+s \Phi_{0}+s^{2} \Psi_{0}+O\left(s^{3}\right),
$$


as $s \simeq 0$ and $\int_{\Omega} \Phi_{0} \Psi_{0}=0$. Moreover, there exists a neighborhood $\mathcal{W}$ of $\left(\lambda_{0}, u_{0}\right)$ in $\mathbb{R} \times P$ such that if $(\lambda, u) \in \mathcal{W}$ is a positive solution to $(1.1)$, then $(\lambda, u)=(\lambda(s), u(s))$ for some $s \in J$. Moreover,

$$
\operatorname{sgn} \lambda^{\prime}(s)=\operatorname{sgn}\left(\Lambda\left(N-p u(s)^{p-1}, N+q u(s)^{q-1}\right)-\lambda(s)\right) .
$$

Proof. Denote

$$
f(\rho)=\frac{1}{\rho}\left(K+\lambda_{0}\right)-K
$$

and

$$
g(\rho)=\Lambda\left(N-\frac{p}{\rho} u_{0}^{p-1}, N+K+\frac{1}{\rho}\left(q u_{0}^{q-1}-K\right)\right) .
$$

By (5.3), it is clear that $f$ is a decreasing function, while $g$ is increasing. If $\left(\lambda_{0}, u_{0}\right)$ is stable, i.e., the first inequality of (5.5) holds, then $g(1)>f(1)$ and so 1 is not an eigenvalue of $\partial_{u} F\left(u_{0}, \lambda_{0}\right)$. Thus $I d-\partial_{u} F\left(u_{0}, \lambda_{0}\right)$ is a topological isomorphism and we can apply Proposition 20.6 of [2] and conclude the first paragraph.

If (5.6) holds, 1 is an eigenvalue of $\partial_{u} F\left(u_{0}, \lambda_{0}\right)$ with a positive eigenfunction, so we can apply Propositions 20.7 and 20.8 of [2]. Finally, observe that from Proposition 20.8 of [2] it follows that

$$
\operatorname{sgn} \lambda^{\prime}(s)=\operatorname{sgn}(1-r(u(s), \lambda(s))) .
$$

Taking into account (5.4) it is not hard to show that

$$
\operatorname{sgn}(1-r(u(s), \lambda(s)))=\operatorname{sgn}\left(\Lambda\left(N-p u(s)^{p-1}, N+q u(s)^{q-1}\right)-\lambda(s)\right) .
$$

This completes the proof.

Proposition 5.6. Assume that $q \leq p$. Let $\left(\lambda_{0}, u_{0}\right)$ be a neutrally stable positive solution to (1.1) with $\lambda=\lambda_{0}$. Then,

$$
\lambda_{2}<0
$$

where $\lambda_{2}$ is defined by (5.8).

Proof. By Lemma 5.5, for $s \in J$, we have

$$
\begin{cases}-\Delta\left(u_{0}+s \Phi_{0}+s^{2} \Psi_{0}+O\left(s^{3}\right)\right)=\lambda_{0} u_{0}+s \lambda_{0} \Phi_{0}+s^{2} \lambda_{0} \Psi_{0}+s^{2} \lambda_{2} u_{0}+O\left(s^{3}\right) & \text { in } \Omega, \\ \frac{\partial\left(u_{0}+s \Phi_{0}+s^{2} \Psi_{0}+O\left(s^{3}\right)\right)}{\partial \nu}=\left(u_{0}+s \Phi_{0}+s^{2} \Psi_{0}+O\left(s^{3}\right)\right)^{p} & \text { on } \Gamma_{1}, \\ \frac{\partial\left(u_{0}+s \Phi_{0}+s^{2} \Psi_{0}+O\left(s^{3}\right)\right)}{\partial \nu}=-\left(u_{0}+s \Phi_{0}+s^{2} \Psi_{0}+O\left(s^{3}\right)\right)^{q} & \text { on } \Gamma_{2} .\end{cases}
$$

After differentiating twice in $s$ and setting $s=0$, we obtain

$$
\begin{cases}-\Delta \Psi_{0}-\lambda_{0} \Psi_{0}=\lambda_{2} u_{0} & \text { in } \Omega, \\ \frac{\partial \Psi_{0}}{\partial \nu}-p u_{0}^{p-1} \Psi_{0}=\frac{p(p-1)}{2} u_{0}^{p-2} \Phi_{0}^{2} & \text { on } \Gamma_{1}, \\ \frac{\partial \Psi_{0}}{\partial \nu}+q u_{0}^{q-1} \Psi_{0}=-\frac{q(q-1)}{2} u_{0}^{q-2} \Phi_{0}^{2} & \text { on } \Gamma_{2} .\end{cases}
$$


Finally, multiplying (5.10) by $\Phi_{0}$, integrating and taking into account (5.7), we obtain

$$
\lambda_{2} \int_{\Omega} u_{0} \Phi_{0}=\frac{1}{2}\left(q(q-1) \int_{\Gamma_{2}} \Phi_{0}^{3} u_{0}^{q-1}-p(p-1) \int_{\Gamma_{1}} \Phi_{0}^{3} u_{0}^{p-1}\right) .
$$

The sign of $\lambda_{2}$ can be determined by Picone's identity, (see Section 4 in [6] and Lemma 4.1 in [26], for instance). Let $u, v \in C^{2}(\Omega) \cap C^{1}(\bar{\Omega})$ be such that $v / u \in C(\bar{\Omega})$ and $\Upsilon:[0, \infty) \mapsto$ $\mathbb{R}$ an arbitrary $C^{1}$ function. Then

$$
\int_{\Omega} \Upsilon\left(\frac{v}{u}\right)(-v \Delta u+u \Delta v)=-\int_{\Omega} \Upsilon^{\prime}\left(\frac{v}{u}\right) u^{2}\left|\nabla\left(\frac{v}{u}\right)\right|^{2}-\int_{\partial \Omega} \Upsilon\left(\frac{v}{u}\right)\left[v \frac{\partial u}{\partial \nu}-u \frac{\partial v}{\partial \nu}\right] .
$$

Observe that

$$
-\Phi_{0} \Delta u_{0}+u_{0} \Delta \Phi_{0}=0
$$

and so taking $\Upsilon(t)=t^{2}, v=\Phi_{0}$ and $u=u_{0}$ in (5.12), we have that

$$
0=-2 \int_{\Omega} \Phi_{0} u_{0}\left|\nabla\left(\frac{\Phi}{u_{0}}\right)\right|^{2}-\int_{\partial \Omega}\left(\frac{\Phi_{0}}{u_{0}}\right)^{2}\left[\Phi_{0} \frac{\partial u_{0}}{\partial \nu}-u_{0} \frac{\partial \Phi_{0}}{\partial \nu}\right]
$$

hence

$$
\int_{\partial \Omega}\left(\frac{\Phi_{0}}{u_{0}}\right)^{2}\left[\Phi_{0} \frac{\partial u_{0}}{\partial \nu}-u_{0} \frac{\partial \Phi_{0}}{\partial \nu}\right]<0
$$

or equivalently,

$$
(q-1) \int_{\Gamma_{2}} u_{0}^{q-2} \Phi_{0}^{3}<(p-1) \int_{\Gamma_{1}} u_{0}^{p-2} \Phi_{0}^{3} .
$$

Since $q \leq p$, by (5.11) we finally deduce that $\lambda_{2}<0$.

As an easy consequence of Lemma 5.5, relation (5.9) and Proposition 5.6, we obtain:

Corollary 5.7. Let $\left(\lambda_{0}, u_{0}\right)$ be a positive neutrally stable solution to (1.1). Then, there exists $\varepsilon>0$ such that for each $\lambda \in\left(\lambda_{0}-\varepsilon, \lambda_{0}\right)$, (1.1) has two positive solutions, one of them stable and the other one unstable. Moreover, there exist a neighborhood $\mathcal{N}$ of $\left(\lambda_{0}, u_{0}\right)$ in $\mathbb{R} \times P$ such that (1.1) does not have positive solutions in $\mathcal{N}$ for $\lambda>\lambda_{0}$.

Finally, we need to prove that any solution to (1.1) is unstable for negative $\lambda$.

Proposition 5.8. Assume that $p \geq q>1$ and let $\left(\lambda_{0}, u_{0}\right)$ be a positive solution to (1.1) with $\lambda_{0} \leq 0$. Then, $\left(\lambda_{0}, u_{0}\right)$ is unstable.

Proof. By (5.5), we have to prove that $\Lambda\left(N-p u_{0}^{p-1}, N+q u_{0}^{q-1}\right)<\lambda_{0}$. For this (see for instance Lemma 2.2 in [16]), it suffices to find a positive function $\underline{u}$ such that

$$
\begin{cases}-\Delta \underline{u} \leq \lambda_{0} \underline{u} & \text { in } \Omega, \\ \frac{\partial \underline{u}}{\partial \nu}-p u_{0}^{p-1} \underline{u} \leq 0 & \text { on } \Gamma_{1}, \\ \frac{\partial \underline{u}}{\partial \nu}+q u_{0}^{q-1} \underline{u} \leq 0 & \text { on } \Gamma_{2},\end{cases}
$$

with some inequality strict (that is $\leq 0$ and $\not \equiv 0$ ). Take $r \in[q, p]$ and consider $\underline{u}=u_{0}^{r}$. Then, in $\Omega$ since $\lambda_{0} \leq 0$ and $r>1$, we get

$$
-\Delta\left(u_{0}^{r}\right)=r(1-r) u_{0}^{r-2}\left|\nabla u_{0}\right|^{2}+r \lambda_{0} u_{0}^{r} \leq \lambda_{0} u_{0}^{r}
$$


while on $\Gamma_{1}$

$$
\frac{\partial u_{0}^{r}}{\partial \nu}-p u_{0}^{p-1} u_{0}^{r}=(r-p) u_{0}^{r+p-1} \leq 0
$$

and finally, on $\Gamma_{2}$,

$$
\frac{\partial u_{0}^{r}}{\partial \nu}+q u_{0}^{q-1} u_{0}^{r}=(q-r) u_{0}^{r+q-1} \leq 0
$$

Hence $u_{0}$ is unstable.

Now, we are ready to prove the uniqueness of stable solution. We use a similar argument to the one used in Theorem 3.7 of [21], see also [11], [12], [23], [24] and [30] where the idea of using the "turning direction" at all possible "turning points" is used.

Proof of Theorem 5.4. We show that the minimal solution $u_{\lambda}$ is stable for all $\lambda \in(0, \Lambda)$. It is well known (see Proposition 20.4 in [2]) that the minimal solution is weakly stable, i.e.,

$$
\Lambda\left(N-p u_{\lambda}^{p-1}, N+q u_{\lambda}^{q-1}\right) \geq \lambda \quad \text { for all } \lambda \in(0, \Lambda) .
$$

On the other hand, in a neighborhood $\mathcal{N}$ of $(\lambda, u)=(0,0)$, there exists a unique positive solution for every $\lambda$. Since the minimal solution exists for all $\lambda \in(0, \Lambda)$, the unique solution coincides with the minimal, so by Corollary 5.7 there exists $\underline{\lambda}$ such that for all $0<\lambda \leq \underline{\lambda}$ we have that $\Lambda\left(N-p u_{\lambda}^{p-1}, N+q u_{\lambda}^{q-1}\right)>\lambda$. Now, we can produce this branch to the right to reach a value $\lambda_{0} \leq \Lambda$ such that $\Lambda\left(N-p u_{\lambda}^{p-1}, N+q u_{\lambda}^{q-1}\right)>\lambda$ for all $\lambda<\lambda_{0}$ and

$$
\Lambda\left(N-p u_{\lambda_{0}}^{p-1}, N+q u_{\lambda_{0}}^{q-1}\right)=\lambda_{0} .
$$

If $\lambda_{0}=\Lambda$ we have finished, so assume that $\lambda_{0}<\Lambda$. By (5.13) and Corollary 5.7, we can always find $\lambda_{1} \in\left(\lambda_{0}, \Lambda\right)$ such that

$$
\Lambda\left(N-p u_{\lambda_{1}}^{p-1}, N+q u_{\lambda_{1}}^{q-1}\right)>\lambda_{1} .
$$

By Lemma 5.5, part 1, we can continue to the left from $u_{\lambda_{1}}$. Denote

$$
\Gamma=\left\{(\lambda, u(\lambda)): \lambda \leq \lambda_{1}\right\} .
$$

Now, two possibilities may occur:

1. There exists $\lambda_{2}<\lambda_{1}$ such that $\Lambda\left(N-p u\left(\lambda_{2}\right)^{p-1}, N+q u\left(\lambda_{2}\right)^{q-1}\right)=\lambda_{2}$.

2. The branch $\Gamma$ can be produced for all $\lambda \leq \lambda_{1}$ with $\Lambda\left(N-p u(\lambda)^{p-1}, N+q u(\lambda)^{q-1}\right)>\lambda$.

If the first possibility holds, then Corollary 5.7 is contradicted. In the second possibility, $\Gamma$ does not reach negative values of $\lambda$ by Proposition 5.8. So, again two possibilities appear:

1. $\Gamma$ meets the real axis $\{(\lambda, 0)\}$.

2. $\Gamma$ degenerates in some minimal solution $u_{\lambda_{3}}$.

If $\Gamma$ degenerates in the axis $\{(\lambda, 0)\}$, since we know that the unique bifurcation point from the trivial solution is $\lambda=0$, then $\Gamma$ degenerates in $(0,0)$. But, it is well known that in a neighborhood $\mathcal{N}$ of $(\lambda, u)=(0,0)$ there exists a unique solution, in fact the minimal solution. So, second possibility occurs. If $\lambda_{3}$ is such that $u_{\lambda_{3}}$ satisfies (5.14), Corollary 5.7 leads to a contradiction. However, if $\lambda_{3}$ is such that $u_{\lambda_{3}}$ satisfies $\Lambda\left(N-p u_{\lambda_{3}}^{p-1}, N+q u_{\lambda_{3}}^{q-1}\right)>$ 
$\lambda_{3}$, we know that in a neighborhood $\mathcal{M}$ of $\left(\lambda_{3}, u_{\lambda_{3}}\right)$ there exists a unique solution, again a contradiction. So, the minimal solution $u_{\lambda}$ is stable for all $\lambda \in(0, \Lambda)$ and neutrally stable for $\lambda=\Lambda$.

Now, assume that for some $\lambda_{0} \in(0, \Lambda)$ there exists a second stable solution $v_{0}>u_{\lambda_{0}}$. We argue as in the first part of the proof. By Lemma 5.5, part 1, there exists a branch, say $\Gamma^{\prime}$, of stable solutions of the form $(\lambda(s), v(s)), s \in I$, with $\lambda(0)=\lambda_{0}, v(0)=v_{0}$. Moreover, we can continue this branch to the left until there exists a value $\lambda^{*}$ in which it is noncontinuable. Since, by Proposition 5.8, all solutions are unstable for $\lambda \leq 0$, it follows that $\lambda^{*} \geq 0$.

If $\lambda^{*}>0$, we would have thanks to Lemma 5.5, part 1, that we arrive at a contradiction with Corollary 5.7. Hence $\lambda^{*}=0$. Moreover, the branch $\Gamma^{\prime}$ has to degenerate at $(0,0)$, otherwise we could continue it thanks to Lemma 5.5, part 1. However, this contradicts the uniqueness of solutions for $\lambda \sim 0$, and the uniqueness of the stable solution is proved.

We finally prove the assertions about $\Lambda$.

Lemma 5.9. If $p<q$ or $p=q$ and $\left|\Gamma_{2}\right|>\left|\Gamma_{1}\right|$, then $\Lambda>0$, while for $p=q$ and $\left|\Gamma_{2}\right| \leq\left|\Gamma_{1}\right|$, we have $\Lambda=0$.

Proof. If $p<q$ or $p=q$ and $\left|\Gamma_{2}\right|>\left|\Gamma_{1}\right|$, the assertion is immediate, since the bifurcation from the trivial solution is to the right, according to Proposition 4.2. Thus assume $p=q$. It is enough to prove that there are no positive solutions to (1.1) for $\lambda=0$.

Assume there exists a positive solution $u$ to (1.1) with $\lambda=0$. If we multiply the equation in (1.1) by $u^{-p}$ and integrate in $\Omega$, we arrive at:

$$
-p \int_{\Omega} u^{-p-1}|\nabla u|^{2}-\left|\Gamma_{1}\right|+\left|\Gamma_{2}\right|=0,
$$

and since $u$ is not constant, we obtain a contradiction. Hence no positive solutions exist for $\lambda=0$, and it follows that $\Lambda=0$.

\subsection{Case III. $p, q<1$}

In this subsection we deal with the case $p, q<1$. Since uniqueness for $p \leq q<1$ and $\lambda \leq 0$ follows from Lemma 2.4, we only need to prove the assertions about existence and nonexistence.

Proof of Theorem 1.3. According to Proposition 4.1, there is a continuum $\mathcal{C}_{\infty}$ of positive solutions bifurcating from $(0,+\infty)$. We are showing next that alternative 2 in Proposition 4.1 holds. For this, it is enough to show that the continuum $\mathcal{C}_{\infty}$ cannot meet the axis $(\lambda, 0)$. Assume on the contrary that there exist solutions $\left(\lambda_{n}, u_{n}\right)$ with $\lambda_{n} \rightarrow \lambda_{0}$ and $\left\|u_{n}\right\|_{\infty} \rightarrow 0$. Then, thanks to the simplicity of principal eigenvalues, $\lambda_{n}=\Lambda\left(N-u_{n}^{p-1}, N+u_{n}^{q-1}\right)$. We now use the properties of this eigenvalue, collected in Proposition 2.1. We have

$$
\Lambda\left(N-u_{n}^{p-1}, N+u_{n}^{q-1}\right)<\Lambda\left(N-u_{n}^{p-1}, D\right),
$$

and since $p<1$ and $u_{n} \rightarrow 0$, we have that this last eigenvalue tends to $-\infty$ as $n \rightarrow \infty$ (cf. Remark 1). This contradiction proves that there exists a solution for all $\lambda \leq 0$. Next, let

$$
\Lambda=\sup \{\lambda \in \mathbb{R}: \text { (1.1) has a positive solution }\} .
$$


It is clear that $0 \leq \Lambda<\infty$, thanks to Lemma 2.2. We are showing now that there exists a solution for all $\lambda<\Lambda$ (of course, this is only to be proved when $\Lambda>0$ ). We use the method of sub and supersolutions.

Thanks to the definition of $\Lambda$, for every $\lambda_{0}<\Lambda$, there exists $\bar{\lambda} \in\left(\lambda_{0}, \Lambda\right)$ such that (1.1) admits a positive solution $\bar{u}$ with $\lambda=\bar{\lambda}$. This solution is clearly a supersolution for (1.1) with $\lambda=\lambda_{0}$.

To obtain a subsolution we note that, by Proposition 2.1 there exists a unique $\eta>0$ such that $\lambda=\Lambda(N-\eta, D)$. Let $\psi$ be an associated normalized positive eigenfunction, i.e., a solution to

$$
\left\{\begin{array}{ccc}
-\Delta \psi=\lambda \psi & \text { in } & \Omega \\
\frac{\partial \psi}{\partial \nu}=\eta \psi & \text { on } & \Gamma_{1} \\
\psi=0 & \text { on } & \Gamma_{2}
\end{array}\right.
$$

and set $\underline{u}=\varepsilon \psi$ for a small positive $\varepsilon$. We claim that $\underline{u}$ is the desired subsolution. Indeed, we only have to check it on $\partial \Omega$. On $\Gamma_{1}$ we get

$$
\frac{\partial \underline{u}}{\partial \nu}=\varepsilon \frac{\partial \psi}{\partial \nu}=\varepsilon \eta \psi \leq \varepsilon^{p} \psi^{p}=\underline{u}^{p}
$$

if $\varepsilon$ is small enough. On $\Gamma_{2}$ we have $\underline{u}=0$, while

$$
\frac{\partial \underline{u}}{\partial \nu}<0
$$

Therefore $\underline{u}$ is a subsolution to (1.1). Whence we obtain a positive solution for $\lambda=\lambda_{0}$.

It is also clear that there exists a solution for $\lambda=\Lambda$, in case $\Lambda>0$. Indeed, let $\lambda_{n} \rightarrow \Lambda$ be an arbitrary sequence, with corresponding solutions $u_{n}$. Since, according to Proposition 4.1, the only point of bifurcation from infinity is $\lambda=0$, we obtain that the sequence $u_{n}$ is bounded. Thus it is standard to pass to the limit to obtain a solution $u_{0}$ to (1.1) with $\lambda=\Lambda$. The same reasoning as in the beginning of the proof shows that $u_{0} \neq 0$, and hence it is a positive solution to (1.1).

We finally remark that the assertions about $\Lambda$ are proved exactly in the same way as in Lemma 5.9, and therefore the proof is omitted.

\subsection{Case IV. $q<1<p$}

We use variational methods in this case to prove the existence of solutions. To this end, let us consider the functional

$$
F(u)=\frac{1}{2} \int_{\Omega}|\nabla u|^{2}-\frac{\lambda}{2} \int_{\Omega}\left|u_{+}\right|^{2}-\frac{1}{p+1} \int_{\Gamma_{1}}\left|u_{+}\right|^{p+1}+\frac{1}{q+1} \int_{\Gamma_{2}}|u|^{q+1}
$$

in $H^{1}(\Omega)$, where $u_{+}=\max \{u, 0\}$ stands for the positive part of $u$. Since $1<p<$ $m /(m-2)$, the functional $F$ is well defined in $H^{1}(\Omega)$. It is moreover $C^{1}$. Our intention is to apply the Mountain Pass Theorem of [4] to $F$. Thus we are seeing that all conditions required are met. We begin with the important Palais-Smale condition.

Lemma 5.10. If $\lambda<\Lambda(N, D)$ then the functional $F$ verifies the Palais-Smale condition. 
Proof. Let $u_{n}$ be a Palais-Smale sequence, that is a sequence such that

$$
\left|F\left(u_{n}\right)\right| \leq C, \quad \text { and } \quad F^{\prime}\left(u_{n}\right) \rightarrow 0 .
$$

We have to prove that it contains a (strongly) convergent subsequence. Let us first check that $\left\{u_{n}\right\}$ is bounded in $H^{1}(\Omega)$.

Assume for a contradiction that this is not the case, that is, passing to a subsequence

$$
\left\|u_{n}\right\|_{H^{1}(\Omega)} \rightarrow \infty .
$$

Let

$$
v_{n}=\frac{u_{n}}{\left\|u_{n}\right\|_{H^{1}(\Omega)}} .
$$

Since $v_{n}$ is bounded in $H^{1}(\Omega)$, and thanks to the compactness of the embeddings $H^{1}(\Omega) \subset$ $L^{2}(\Omega), H^{1}(\Omega) \subset L^{p+1}\left(\Gamma_{1}\right)$ and $H^{1}(\Omega) \subset L^{q+1}\left(\Gamma_{2}\right)$, there exists a subsequence (that we still denote by $\left.v_{n}\right)$ such that

$$
\begin{aligned}
& v_{n} \rightarrow v_{0} \\
& v_{n} \rightarrow v_{0} \quad \text { weakly in } H^{1}(\Omega), \\
& \text { strongly in } L^{2}(\Omega), L^{p+1}\left(\Gamma_{1}\right), L^{q+1}\left(\Gamma_{2}\right),
\end{aligned}
$$

for a certain $v_{0} \in H^{1}(\Omega)$.

On the other hand since $v_{n}$ is bounded in $H^{1}(\Omega)$, we have

$$
\begin{aligned}
\frac{F\left(u_{n}\right)}{\left\|u_{n}\right\|_{H^{1}(\Omega)}}- & \frac{1}{2}\left\langle F^{\prime}\left(u_{n}\right), v_{n}\right\rangle \\
= & \left(\frac{1}{2}-\frac{1}{p+1}\right)\left\|u_{n}\right\|_{H^{1}(\Omega)}^{p} \int_{\Gamma_{1}}\left|\left(v_{n}\right)_{+}\right|^{p+1} \\
& +\left(\frac{1}{q+1}-\frac{1}{2}\right)\left\|u_{n}\right\|_{H^{1}(\Omega)}^{q} \int_{\Gamma_{2}}\left|v_{n}\right|^{q+1} \rightarrow 0 .
\end{aligned}
$$

Hence

$$
\left\|u_{n}\right\|_{H^{1}(\Omega)}^{p} \int_{\Gamma_{1}}\left|\left(v_{n}\right)_{+}\right|^{p+1} \rightarrow 0,
$$

and

$$
\left\|u_{n}\right\|_{H^{1}(\Omega)}^{q} \int_{\Gamma_{2}}\left|v_{n}\right|^{q+1} \rightarrow 0 .
$$

By the strong convergence of $v_{n}$ to $v_{0}$ in $L^{q+1}\left(\Gamma_{2}\right)$, it follows in particular that $v_{0}=0$ on $\Gamma_{2}$ (in the sense of traces). Furthermore, since $\left\langle F^{\prime}\left(u_{n}\right), v_{n}\right\rangle \rightarrow 0$, we also have

$$
\begin{aligned}
\left\|u_{n}\right\|_{H^{1}(\Omega)} & \left(\int_{\Omega}\left|\nabla v_{n}\right|^{2}-\lambda \int_{\Omega}\left|\left(v_{n}\right)_{+}\right|^{2}\right)-\left\|u_{n}\right\|_{H^{1}(\Omega)}^{p} \int_{\Gamma_{1}}\left|\left(v_{n}\right)_{+}\right|^{p+1} \\
& +\left\|u_{n}\right\|_{H^{1}(\Omega)}^{q} \int_{\Gamma_{2}}\left|v_{n}\right|^{q+1} \rightarrow 0 .
\end{aligned}
$$

Thus thanks to (5.15) and (5.16) we have that

$$
\int_{\Omega}\left|\nabla v_{n}\right|^{2}-\lambda \int_{\Omega}\left|\left(v_{n}\right)_{+}\right|^{2} \rightarrow 0 .
$$


By lower semicontinuity, it follows that

$$
\int_{\Omega}\left|\nabla v_{0}\right|^{2}-\lambda \int_{\Omega}\left|\left(v_{0}\right)_{+}\right|^{2} \leq 0,
$$

and since $v_{0}=0$ on $\Gamma_{2}$ and $\lambda<\Lambda(N, D)$, the variational characterization of this eigenvalue implies that $v_{0}=0$ in $\Omega$. Hence by (5.17) we have

$$
\int_{\Omega}\left|\nabla v_{n}\right|^{2} \rightarrow 0
$$

which contradicts $\left\|v_{n}\right\|_{H^{1}(\Omega)}=1$. This proves that $u_{n}$ is bounded in $H^{1}(\Omega)$.

Next we prove that $\left\{u_{n}\right\}$ contains a strongly convergent subsequence. Since $u_{n}$ is bounded in $H^{1}(\Omega)$ there exists a subsequence (that we denote again by $u_{n}$ ) such that

$$
\begin{array}{ll}
u_{n} \rightarrow u_{0} & \text { weakly in } H^{1}(\Omega), \\
u_{n} \rightarrow u_{0} & \text { strongly in } L^{2}(\Omega), L^{p+1}\left(\Gamma_{1}\right), L^{q+1}\left(\Gamma_{2}\right) .
\end{array}
$$

Since $\left\langle F^{\prime}\left(u_{n}\right), u_{n}\right\rangle \rightarrow 0,\left\langle F^{\prime}\left(u_{n}\right), u_{0}\right\rangle \rightarrow 0$, we obtain

$$
\lim _{n \rightarrow \infty} \int_{\Omega}\left|\nabla u_{n}\right|^{2}=\lambda \int_{\Omega}\left|\left(u_{0}\right)_{+}\right|^{2}-\int_{\Gamma_{1}}\left|\left(u_{0}\right)_{+}\right|^{p+1}+\int_{\Gamma_{2}}\left|u_{0}\right|^{q+1},
$$

while

$$
\int_{\Omega}\left|\nabla u_{0}\right|^{2}=\lambda \int_{\Omega}\left|\left(u_{0}\right)_{+}\right|^{2}-\int_{\Gamma_{1}}\left|\left(u_{0}\right)_{+}\right|^{p+1}+\int_{\Gamma_{2}}\left|u_{0}\right|^{q+1},
$$

thanks to weak convergence. From (5.18) and (5.19) we obtain

$$
\lim _{n \rightarrow \infty} \int_{\Omega}\left|\nabla u_{n}\right|^{2}=\int_{\Omega}\left|\nabla u_{0}\right|^{2}
$$

and we conclude that $u_{n}$ converges to $u_{0}$ strongly in $H^{1}(\Omega)$.

Now we prove that the functional $F$ verifies the geometric hypotheses required to apply the mountain pass theorem.

Lemma 5.11. Let $\lambda<\Lambda(N, D)$. Then the functional $F$ verifies:

1. There exists a positive constant $c$ such that for small enough $r, F(u) \geq c r^{3}$ if $\|u\|_{H^{1}(\Omega)}=r$.

2. There exists $v_{0}$ with large $H^{1}(\Omega)$-norm such that $F\left(v_{0}\right)<0$.

Proof. 1. We argue by contradiction. Assume that there exists a sequence $u_{n}$ such that

$$
\left\|u_{n}\right\|_{H^{1}(\Omega)}=r_{n} \rightarrow 0, \quad \text { and } \quad \frac{F\left(u_{n}\right)}{r_{n}^{3}} \rightarrow 0 .
$$

Let $v_{n}=u_{n} / r_{n}$. Since $\left\|v_{n}\right\|_{H^{1}(\Omega)}=1$ we can extract a subsequence such that

$$
\begin{aligned}
& v_{n} \rightarrow v_{0} \\
& v_{n} \rightarrow v_{0} \quad \text { weakly in } H^{1}(\Omega), \\
& \text { strongly in } L^{2}(\Omega), L^{p+1}\left(\Gamma_{1}\right), L^{q+1}\left(\Gamma_{2}\right) .
\end{aligned}
$$


From (5.20) we obtain

$$
\int_{\Omega}\left|\nabla v_{n}\right|^{2}-\lambda \int_{\Omega}\left|\left(v_{n}\right)_{+}\right|^{2}-r_{n}^{p-1} \int_{\Gamma_{1}}\left|\left(v_{n}\right)_{+}\right|^{p+1}+r_{n}^{q-1} \int_{\Gamma_{2}}\left|v_{n}\right|^{q+1} \rightarrow 0 .
$$

Hence the weak limit $v_{0}$ verifies

$$
\int_{\Omega}\left|\nabla v_{0}\right|^{2}-\lambda \int_{\Omega}\left|\left(v_{0}\right)_{+}\right|^{2} \leq 0, \quad v_{0}=0 \text { on } \Gamma_{2} .
$$

As $\lambda<\Lambda(N, D)$ we get $v_{0} \equiv 0$, but in this case from (5.21) we get

$$
\int_{\Omega}\left|\nabla v_{n}\right|^{2} \rightarrow 0
$$

a contradiction with $\left\|v_{n}\right\|_{H^{1}(\Omega)}=1$.

2. We take any function $v$ such that $v \neq 0$ on $\Gamma_{1}$ and observe that

$$
\lim _{t \rightarrow \infty} F(t v)=-\infty
$$

Thus it suffices with setting $v_{0}=t v$ for a large enough $t$.

Proof of Theorem 1.4. To prove existence of solutions when $\lambda<\Lambda(N, D)$, we just have to use the mountain pass theorem (see [4]), since in this range of $\lambda$ 's the functional $F$ verifies the Palais-Smale condition thanks to Lemma 5.10 and the geometric hypothesis needed to apply it are guaranteed by Lemma 5.11.

Thus the functional $F$ has a nontrivial critical point $u$, which is a weak solution to

$$
\left\{\begin{aligned}
-\Delta u & =\lambda u_{+} & & \text {in } \Omega, \\
\frac{\partial u}{\partial \nu} & =\left(u_{+}\right)^{p} & & \text { on } \Gamma_{1} \\
\frac{\partial u}{\partial \nu} & =-|u|^{q-1} u & & \text { on } \Gamma_{2} .
\end{aligned}\right.
$$

Taking $u_{-}=\min \{u, 0\}$ as a test function in the weak formulation of (5.22) it follows that $u \geq 0$. Thus by the strong maximum principle and Hopf's Lemma we have that $u$ is a positive weak solution to (1.1). According to regularity theory, $u \in C^{2, \alpha}(\bar{\Omega}) \cap C^{\infty}(\Omega)$.

To finish the proof, we just notice that Lemma 2.2 implies that there are no nontrivial solutions for $\lambda \geq \Lambda(N, D)$.

5.5 Case V. $q=1>p, q=1<p, p=1>q, p=1<q$

In this section we deal with the borderline cases in which one of the powers is equal to one. We sketch very briefly the main arguments.

\subsubsection{Case $q=1>p$.}

We remark first that the uniqueness of solutions is a consequence of Lemma 2.3. Thus, we only consider the existence issue. 
By proceeding as in the proof of Lemma 2.2 , it is easily shown that there are no positive solutions for $\lambda \geq \Lambda(N, N+1)$. Indeed, if $\phi$ is a positive eigenfunction associated to this eigenvalue, and we multiply the equation in (1.1) by $\phi$ and integrate in $\Omega$, we arrive at

$$
(\Lambda(N, N+1)-\lambda) \int_{\Omega} u \phi=\int_{\Gamma_{1}} \phi u^{p} .
$$

Hence, no positive solutions exist for $\lambda \geq \Lambda(N, N+1)$.

To show the existence for $\lambda<\Lambda(N, N+1)$, we use the method of sub and supersolutions. The proof is similar to that of Theorem 1.1, with slight differences. We choose $\eta>0$ such that $\lambda=\Lambda(N-\eta, N+1)$ (this is possible thanks to Proposition 2.1), and $\phi$ a positive associated eigenfunction, i. e. a solution to

$$
\left\{\begin{aligned}
-\Delta \phi=\lambda \phi \quad & \text { in } \quad \Omega, \\
\frac{\partial \phi}{\partial \nu}=\eta \phi \quad & \text { on } \quad \Gamma_{1} \\
\frac{\partial \phi}{\partial \nu}=-\phi & \text { on } \quad \Gamma_{2}
\end{aligned}\right.
$$

It is not hard to check that $\underline{u}=\varepsilon \phi, \bar{u}=M \phi$ are a pair of sub and supersolutions with $\underline{u} \leq \bar{u}$ in $\Omega$, provided $\varepsilon$ is chosen small enough and $M$ large enough. This shows the existence of a positive solution. As remarked before, it is unique.

\subsubsection{Case $q=1<p$.}

It follows exactly as in the previous subsection that $\lambda<\Lambda(N, N+1)$ is a necessary condition to have a positive solution. To show existence, we note that Proposition 4.2 implies the existence of a continuum $\mathcal{C}_{0}$ of positive solutions emanating from $(\lambda, u)=$ $(\Lambda(N, N+1), 0)$. Moreover, thanks to Lemma 3.4, the solutions are bounded in bounded intervals of $\lambda$. Thus, the existence of positive solutions for all $\lambda<\Lambda(N, N+1)$ follows.

\subsubsection{Case $p=1>q$.}

It is easily seen again that $\Lambda(N-1, N)<\lambda<\Lambda(N-1, D)$ is necessary for the existence of positive solutions to (1.1) (just multiply by a positive eigenfunction $\phi$ associated to $\Lambda(N-1, N)$ or $\Lambda(N-1, D)$ and integrate in $\Omega)$.

To show existence, we apply Proposition 4.1 to get a continuum $\mathcal{C}_{\infty}$ of positive solutions to (1.1) emanating from infinity at the eigenvalue $\lambda=\Lambda(N-1, N)$. By Proposition 4.1 we deduce that $\mathcal{C}_{\infty}$ meets the set $\{(\lambda, 0): \lambda \in \mathbb{R}\}$. So, assume that there exists a sequence $\left(\lambda_{n}, u_{n}\right)$ such that $\lambda_{n} \rightarrow \lambda_{0}$ and $\left\|u_{n}\right\|_{\infty} \rightarrow 0$ as $n \rightarrow \infty$. Since $u_{n}$ is positive, it follows that $\lambda_{n}=\Lambda\left(N-1, N+u_{n}^{q-1}\right) \rightarrow \Lambda(N-1, D)$. This shows that a positive solution exists for every $\lambda \in(\Lambda(N-1, N), \Lambda(N-1, D))$.

\subsubsection{Case $p=1<q$.}

The proof that $\lambda \in(\Lambda(N-1, N), \Lambda(N-1, D))$ is a necessary condition for existence can be obtained exactly as in the previous case. Also, uniqueness is a consequence of Lemma 2.3. Thus we only show existence.

To this aim we employ again the method of sub and supersolutions. Since $\lambda \in(\Lambda(N-$ $1, N), \Lambda(N-1, D)$ ), there exists $\rho>0$ such that $\lambda=\Lambda(N-1, N+\rho)$ (cf. Proposition 
2.1). Let $\phi$ be a positive eigenfunction associated to $\Lambda(N-1, N+\rho)$. Then, it can be easily checked that $\underline{u}=\varepsilon \phi$ is subsolution of (1.1) with a small $\varepsilon$, while $\bar{u}=M \phi$ is supersolution if $M$ is large enough. Therefore the existence of a positive solution for every $\lambda \in(\Lambda(N-1, N), \Lambda(N-1, D))$ follows.

Acknowledgements. Supported by MEC and FEDER under grants MTM2005-06480 (JGM \& JDR), BFM 2003-06446 and MTM2006-07932 (AS), and ANPCyT PICT No. 03-05009 (JDR). JDR is a member of CONICET. Part of this work was done during a visit of JGM and JDR to Univ. de Sevilla. They want to thank for the warm hospitality found there.

\section{References}

[1] H. Amann, Nonlinear elliptic equations with nonlinear boundary conditions, In "New Developments in differential equations" (W. Eckhaus, ed.), Math Studies 21, North-Holland, Amsterdam (1976), 43-63.

[2] H. Amann, Fixed points equations and nonlinear eigenvalue problems in ordered Banach spaces, SIAM Review 18 (1976), 620-709.

[3] H. Amann and J. López-Gómez, A priori bounds and multiple solutions for superlinear indefinite elliptic problems, J. Differential Equations 146 (1998), 336-374.

[4] A. Ambrosetti And P.H. Rabinowitz, Dual variational methods in critical point theory and applications, J. Functional Analysis 14 (1973), 349-381.

[5] J. M. Arrieta, R. Pardo And A. Rodríguez-Bernal, Bifurcation and stability of equilibria with asymptotically linear boundary conditions at infinity, to appear in Proc. Royal Soc. Edinburgh A.

[6] H. Berestycki, I. Capuzzo-Dolcetta and L. Nirenberg, Variational methods for indefinite superlinear homogeneous elliptic problems, NoDEA Nonlinear Differential Equations Appl. 2 (1995), 553-572.

[7] H. Brezis And L. Oswald, Remarks on sublinear elliptic equations, Nonlinear Anal. 10 (1986), 55-64.

[8] S. Cano-Casanova and J. López-Gómez, Continuous dependence of principal eigenvalues with respect to perturbations of the domain around its Dirichlet boundary, Nonlinear Anal. 47 (2001), 1797-1808.

[9] P. Cherrier, Problèmes de Neumann non linéaires sur les variétés Riemanniennes, J. Functional Analysis 57 (1984), 154-206.

[10] M. G. Crandall and P. H. Rabinowitz, Bifurcation, perturbation of simple eigenvalues and linearized stability, Arch. Rational Mech. Anal. 52 (1973), 161-180.

[11] Y. DU, Uniqueness, multiplicity and stability for positive solutions of a pair of reaction-diffusion equations, Proc. Roy. Soc. Edinburgh Sect. A 126 (1996), 777-809. 
[12] Y. Du, Exact multiplicity and S-shaped bifurcation curve for some semilinear elliptic problems from combustion theory, SIAM J. Math. Anal. 32 (2000), 707-733.

[13] J. F. Escobar, Uniqueness theorems on conformal deformations of metrics, Sobolev inequalities, and an eigenvalue estimate, Comm. Pure Appl. Math. 43 (1990), 857883.

[14] J. F. Escobar, Conformal deformation of a Riemannian metric to a scalar flat metric with constant mean curvature, Ann. of Math. (2) 136 (1992), 1-50.

[15] R. Ferreira, F. Quirós And J. D. Rossi, The balance between nonlinear inwards and outwards boundary-flux for a nonlinear heat equation, J. Differential Equations 184 (2002), 259-282.

[16] J. M. Fraile, P. Koch-Medina, J. López-Gómez and S. Merino, Elliptic eigenvalue problems and unbounded continua of positive solutions of a semilinear elliptic equations, J. Differential Equations 127 (1996), 295-319.

[17] J. García-Melián, J. D. Rossi and J. Sabina de Lis, A bifurcation problem governed by the boundary condition II, Proc. London Math. Soc. 94 (2007), 1-25.

[18] J. García-Melián, J. D. Rossi and J. Sabina De Lis, Existence and uniqueness of positive solutions to elliptic problems with nonlinear mixed boundary conditions, preprint.

[19] B. Gidas And J. Spruck, A priori bounds for positive solutions of nonlinear elliptic equations, Comm. Partial Differential Equations 6 (1981), 883-901.

[20] D. Gilbarg And N.S. Trudinger, Elliptic partial differential equations of second order, Springer-Verlag, 1983.

[21] R. Gómez-ReÑAsco And J. López-Gómez, The effect of varying coefficients on the dynamics of a class of superlinear indefinite reaction-diffusion equations, J. Differential Equations 167 (2000), 36-72.

[22] B. Hu, Nonexistence of a positive solution of the Laplace equation with a nonlinear boundary condition, Diff. Int. Eq. 7 (1994), 301-313.

[23] P. Korman, Y. Li And T.OuYAng, Exact multiplicity results for boundary value problems with nonlinearities generalising cubic, Proc. Roy. Soc. Edinburgh Sect. A 126 (1996), 599-616.

[24] P. Korman, Y. Li and T.Ouyang, An exact multiplicity result for a class of semilinear equations, Comm. Partial Differential Equations 22 (1997), 661-684.

[25] O. A. Ladyzhenskaya and N. N. Uralt'seva, Linear and quasilinear elliptic equations, Academic Press, New York, 1968.

[26] J. LóPEZ-GómEz, On the existence of positive solutions for some indefinite superlinear elliptic problems, Comm. Partial Differential Equations 22 (1997), 1787-1804.

[27] F. J. Mancebo And J. M. Vega, A model of porous catalyst accounting for incipiently non-isothermal effects, J. Differential Equations, 151 (1999), 79-110. 
[28] C. Morales-Rodrigo And A. SuÁrez, Some elliptic problems with nonlinear boundary conditions, in "Spectral Theory and Nonlinear Analysis with Applications to Spatial Ecology" (S. Cano-Casanova, J. López-Gómez, C. Mora-Corral, eds.), World Scientific, (2005), 175-199.

[29] C. Morales-Rodrigo ANd A. SuÁrez, Uniqueness of solution for elliptic problems with nonlinear boundary conditions, Comm. Appl. Nonlinear Anal. 13 (2006), 69-78.

[30] T. Ouyang And J. Shi, Exact multiplicity of positive solutions for a class of semilinear problems, Parts I and II, J. Differential Equations 146 (1998), 121-156 and 158 (1999), 94-151.

[31] J. D. Rossi, Elliptic problems with nonlinear boundary conditions and the Sobolev trace theorem, Handbook of Differential Equations: Stationary Partial Differential Equations, Vol. 2. Chapter 5 (M. Chipot, P. Quittner, eds). Elsevier (2005), 311-406.

[32] K. Umezu, Bifurcation from infinity for asymptotically linear elliptic eigenvalue problems, J. Math. Anal. Appl. 267 (2002), 651-664. 